\title{
Anatomia endoscópica do seio esfenoidal
} Endoscopic
anatomy of
the sphenoid sinus

\author{
Alexandre A. Murta', Christiano G. Carneiro², \\ Alexandre Felippu ${ }^{3}$
}

Palavras-chave: seio esfenoidal, cirurgia endoscópica, artéria carótida interna, nervo maxilar, nervo óptico, anatomia.

Key words: sphenoid sinus, endoscopic sinus surgery, internal carotid artery, maxillary nerve, optic nerve, anatomy.

\section{Resumo / Summary}

ntroduçaõ: A importância da compreensão da anatomia interna do seio esfenoidal deve-se à sua peculiar localização no centro do crânio, com estruturas vizinhas de nobreza indiscutível, e por transparecer estas relações anatômicas em suas delgadas paredes internas. Forma de estudo: Anatômico. Material emétodo: No presente estudo foram dissecados endoscopicamente 52 seios esfenoidais, sendo suas saliências e depressões internas analisadas, correspondentes aos relevos internos produzidos pela artéria carótida interna, nervo óptico, nervo maxilar, e nervo vidiano. Resultado: Em 88,5\% dos casos a artéria carótida interna apresentou-se saliente, e o nervo óptico o fez em 55,8\%. 0 nervo vidiano e o nervo maxilar mostraram-se visíveis em $25 \%$ e $30,8 \%$ dos casos, respectivamente. Conclusão: Tais dados nos chamam a atenção para a rica e frágil anatomia interna do seio esfenoidal que, devido ao avanço dos métodos de cirurgia endoscópica, está progressivamente mais próxima e vulnerável a manobras e procedimentos cirúrgicos. Assim, sua compreensão torna-se essencial quando se trata de cirurgia endoscópica endonasal e seu horizonte de recursos técnicos. ntroduction: The internal anatomy of the sphenoid sinus plays a role of great importance due to its peculiar placement in the center of the head, surrounded by important adjacent structures, which make themselves transparent in its internal walls. Study design: Anatomic. Material and method: In this study, 52 sphenoid sinus were endoscopically dissected, and the elevations and depressions presented on its internal walls, produced by the internal carotid artery and the optic, maxillary and vidian nerves, were analyzed. Results: In $88,5 \%$ of the cases, the internal carotid artery were projected into the sinus, while the optic nerve were in $55,8 \%$. The vidian and maxillary nerves were salient in $25 \%$ and $30 \%$ respectively. Conclusion: These data point us to the rich end delicate internal anatomy of the sphenoid sinus, which is progressively closer and more vulnerable to surgical maneuvers, due to the advance of surgical skills, techniques and knowledge. Thus, the study and comprehension of the sphenoid sinus internal anatomy became essential, concerning the endoscopic sinus surgery and its huge field of technical resources.

\footnotetext{
${ }^{1}$ Responsável pelo Setor de Cirurgia Plástica Facial do Instituto Felippu de Rinologia.

${ }^{2}$ Responsável pelo Setor de Laringologia e Voz do Instituto Felippu de Rinologia.

${ }^{3}$ Diretor Geral do Instituto Felippu de Rinologia.

Instituição de O rigem: Instituto Felippu de Rinologia

Endereço para correspondência: Alexandre A. Murta - Rua Stela Marina, 46

Brooklin Novo - 04601-090 - São Paulo - SP

Fone: (0xx11)5536.5353 Fax: (0xx11) 5535.7525

E-mail: alexandremurta@hotmail.com

Artigo recebido em 09 de agosto de 2001. Artigo aceito em 09 de maio de 2002.
} 


\section{INTRODUÇÃO}

O seio esfenoidal apresenta a peculiaridade de refletir suas relações anatômicas em suas paredes ${ }^{1}$. As estruturas vizinhas, já presentes antes do seu desenvolvimento, imprimem um relevo em suas paredes internas na medida em que o seio se pneumatiza e se expande, aumentando seu contato com tais estruturas, ou mesmo englobando-as ${ }^{2,3}$. Assim, à visão endoscópica, nota-se uma cavidade cujas paredes internas mostram-se marcadas por uma série variável de saliências e depressões correspondentes às impressões dos elementos anatômicos vizinhos ${ }^{4}$, sendo que a espessura da lâmina óssea que os separa pode variar de compacta até muito delgada ${ }^{3}$, ou mesmo inexistente ${ }^{5}$. Essa íntima relação contribui para a inquestionável importância clínica e cirúrgica do seio esfenoidal ${ }^{6}$.

As estruturas que usualmente fazem parte desta relação são os vasos e nervos que transitam ao lado do seio, em seus trajetos de aferência e eferência do crânio, sendo as mais freqüentes e importantes a artéria carótida interna e os nervos óptico, maxilar e vidiano (nervo do canal pterigoídeo)?

0 presente estudo tem por finalidade quantificar a intensidade e freqüência em que tais estruturas se projetam no interior do seio esfenoidal, e ressaltar a importância do seu conhecimento anatômico preciso na prática cirúrgica e clínica.

\section{MATERIAL E MÉTODO}

Foram submetidos à dissecção cirúrgica videoendoscópica 52 cadáveres frescos, não-formolizados, adultos, no Programa de Dissecção Cadavérica do Instituto Felippu de Rinologia (São Paulo), tomados randomicamente, e estudados seguindo um mesmo protocolo cirúrgico.

Em cada cadáver, após adequada etmoidectomia, procedeu-se a abertura ampla da parede anterior do seio esfenoidal, permitindo total visualização da cavidade sinusal e do relevo impresso em suas paredes internas. As elevações e recessos foram identificados e os elementos adjacentes comprovados pela delicada remoção das paredes ósseas.

Todas as dissecções foram realizadas com instrumental de cirurgia endoscópica convencional, com telescópios de 30 graus e eventualmente de 70 graus, e gravadas em vídeo para documentação e análise posterior.

\section{RESULTADOS}

Foram dissecados 52 seios esfenoidais e analisadas as saliências e depressões produzidas pela artéria carótida interna e nervos óptico, maxilar e vidiano no relevo de suas paredes internas.

A artéria carótida interna apresentou elevação identificável em 46 (88,5\%) casos, sendo que em 32 (61,5\%) sua projeção foi considerada pronunciada, e em $8(15,4 \%)$ casos to do o seu trajeto serpinginoso, o sifão carotídeo, foi caracterizado. Não foi encontrado nenhum caso de deiscência.

0 nervo óptico foi identificado em 29 (55,8\%) cadáveres. Vale ressaltar que em todos os casos nos quais 0 nervo apresentou saliência na parede do seio, também 0 fez a artéria carótida interna.

0 nervo vidiano (nervo do canal pterigoídeo) mostrou-se visível no assoalho do seio em 13 (25\%) casos, variando desde uma discreta elevação até uma trabécula óssea.

O nervo maxilar projetou-se na parede lateral do seio esfenoidal em $16(30,8 \%)$ casos, muitos deles com a presença concomitante do nervo vidiano abaixo dele.

\section{DISCUSSÃO}

Com o aprimoramento das técnicas de diagnóstico por imagem e de cirurgia endonasal, a anatomia dos seios paranasais e a base anterior do crânio passaram a representar um papel de crescente destaque. Neste contexto, 0 seio esfenoidal assume posição de evidência devido à sua localização singular, praticamente no centro do crânio, em contato íntimo com estruturas nobres, tais como os seis primeiros pares cranianos, a artéria carótida interna, a hipófise, o seio cavernoso, a fossa pterigopalatina e o próprio encéfalo ${ }^{3}$. 0 progressivo avanço da cirurgia endonasal realçou a importância do conhecimento dessa anatomia, a qual passou a ser vista sob outro prisma - o endoscópico ${ }^{8}$. Com 0 auxílio do endoscópio, o cirurgião pode visualizar o interior do seio esfenoidal com grande precisão e proximidade, dispondo de recursos para alterar seu ângulo de visão, permitindo-o "olhar" para os lados, para cima, ou mesmo para frente, através de telescópios de diferentes angulações ${ }^{8}$. Além disso, a cirurgia pode extrapolar o limite de suas barreiras ósseas, transformando o seio esfenoidal em via de acesso para procedimentos neurocirúrgicos, como para a hipófise e certos tumores intracranianos ${ }^{9,10}$. Entretanto, sua

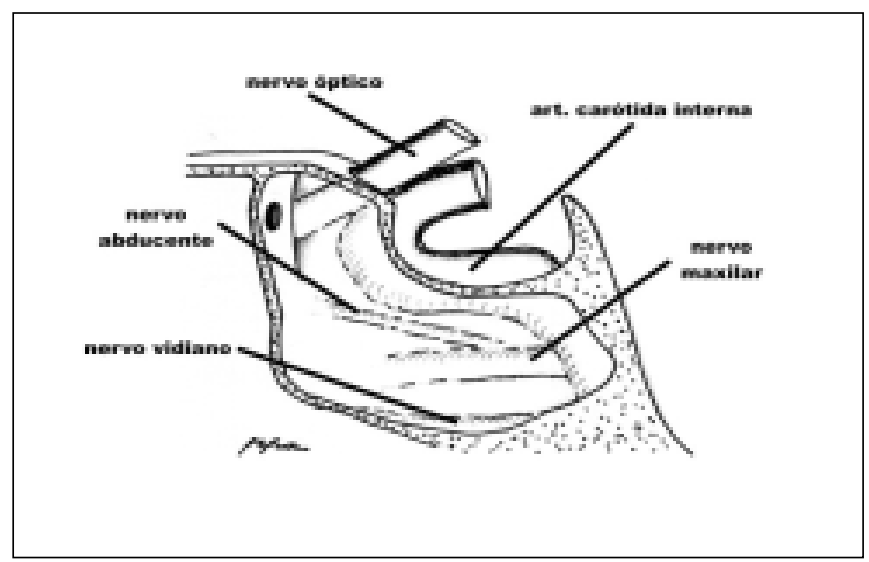

Figura 1. Anatomia seio esfenoidal 
incrível diversidade anatômica caracteriza as inúmeras variações como uma constante, conferindo um valor extra ao seu estudo e conhecimento ${ }^{3}$.

Analisamos o comportamento das principais estruturas relacionadas ao seio esfenoidal - artéria carótida interna e nervos óptico, maxilar e vidiano - em 52 cadáveres frescos, submetidos a dissecção endoscópica.

A artéria carótida interna lidera o grupo, não apenas pela freqüência em que sua elevação aparece $(88,5 \%)$, como também pelo grau de projeção dentro do seio, visto que em $61,5 \%$ dos casos seu relevo foi considerado pronunciado, e em $15,4 \%$ todo o seu trajeto apresentou-se saliente. São incidências mais elevadas que as encontradas por van Alyea ${ }^{6}$, que notou a presença da artéria em $65 \%$ dos casos, sendo que em $53 \%$ a condição foi considerada pronunciada, e em $14 \%$ apresentou seu trajeto completo. Fujii et al. ${ }^{5}$ também estudaram o trajeto da artéria no seio esfenoidal, com resultados semelhantes, além de mencionarem um caso de deiscência do vaso. É inquestionável a importância dessas constatações sob o ponto de vista cirúrgico. 0 conhecimento das variações anatômicas da artéria carótida interna é fundamental para se evitar sua manipulação inadvertida, que pode resultar em sua rotura, uma complicação gravíssima, de difícil controle, com prognóstico sombrio. Diversos autores relatam lesão na artéria carótida durante procedimentos de cirurgia endoscópica endonasal ${ }^{11}$. Após identificada, a hemorragia deve ser imediatamente controlada com tamponamento compressivo, seguido da angiografia de urgência com oclusão permanente por balão. Ocasionalmente, a artéria carótida interna precisa ser sacrificada por ligadura arterial no pescoço, com alto risco de danos neurológicos irreversíveis ${ }^{8}$. Felippu relatou dois casos de lesão transoperatória da artéria carótida intracavernosa no seio esfenoidal controlados com sucesso por fixação de enxerto muco-periostal livre de corneto inferior e curativo compressivo ${ }^{12}$. Maniglia et al. ${ }^{13}$ mencionam três casos de lesão arterial resultando em morte.

Também observamos um número mais elevado de proeminência do nervo óptico (55,8\%), que outros autores, como no estudo de van Alyea ${ }^{6}$, que encontrou $40 \%$ dos casos, ou Lang com $42,85 \% \%^{14}$. A relação entre o nervo óptico e o seios posteriores despertou o interesse de inúmeros autores. Onodi ${ }^{15}$ classificou essa relação em 12 grupos, com 38 variedades anatômicas. Mellinger ${ }^{16}$ ressaltou a importância da assimetria dos seios posteriores em relação à vulnerabilidade do nervo óptico. Ambos, entre muitos outros autores, acreditaram que a projeção do nervo óptico para o interior do seio esfenoidal deveria ser considerada como possível fator etiológico em casos de neurite óptica ${ }^{17}$ e outras doenças órbito-oculares ${ }^{18}$. Essa íntima relação do nervo óptico com o seio esfenoidal e seio etmoidal atribui-Ihe grande vulnerabilidade durante as cirurgias dos seios paranasais. Maniglia et al. relataram uma série de 10 casos de cegueira como complicação maior pós-operatória ${ }^{13}$.
Em nosso estudo, o nervo vidiano (nervo do canal pterigoídeo) mostrou-se saliente em $25 \%$ dos casos, menos freqüentes que quando comparado aos achados de outros autores, como $48 \%{ }^{28}$ e $52 \%{ }^{19}$, ou mesmo índices de $10 \%$ de deiscência do canal pterigoídeo dentro do seio esfenoidal ${ }^{19}$. Zuckerkand ${ }^{20}$, em 1893, foi um dos primeiros a identificar a saliência do nervo vidiano no assoalho do seio, sendo que Sluder ${ }^{21,22}$ e Vai ${ }^{23}$ enfatizaram sua importância clínica. Este último concluiu que a irritação do nervo vidiano produz os sintomas da síndrome de Sluder (do gânglio esfenopalatino), que consiste em rinorréia e dor no assoalho do nariz, no olho ou atrás dele, maxilar e dentes.

0 nervo maxilar, segunda divisão do quinto par craniano, freqüentemente produz uma elevação na parede lateral do seio esfenoidal no seu trajeto no interior do seio cavernoso, antes de atingir o forame redondo ${ }^{24,25}$, fato esse observado em $40 \%$ dos casos segundo van Alyea ${ }^{6}$, em comparação com os $30,8 \%$ por nós identificados, e $28,6 \%$ por Lang ${ }^{19}$. Houser ${ }^{26}$ concluiu que essa proximidade anatômica pode afetar o nervo maxilar em casos de empiema do seio esfenoidal, com neurite, mesmo estando o nervo mergulhado em sangue do seio cavernoso. Nessa mesma linha, Garber ${ }^{27}$ chamou a atenção para o fato de que o nervo maxilar está tão próximo ao seio esfenoidal quanto o nervo vidiano, estando assim também implicado na síndrome do gânglio esfenopalatino. Sluder ${ }^{28}$ reforçou esse argumento demonstrando a permeabilidade das paredes do seio esfenoidal através da injeção de cocaína em seu interior, provocando a paralisia do nervo maxilar.

Embora não seja objetivo deste estudo, ressaltamos que o seio esfenoidal apresenta um conjunto muito rico de variações anatômicas em relação aos seios etmoidais posteriores, à sela túrcica, à fossa pterigopalatina, ao seio cavernoso, bem como diferentes morfologias de recessos, pneumatização e simetria ${ }^{29,30}$.

\section{CONCLUSÃO}

Com o presente estudo, demonstramos a complexidade da anatomia do seio esfenoidal e de suas relações com estruturas nobres, bem como sua ampla gama de variações. Salientamos a importância da compreensão desta anatomia para sua adequada interpretação clínica e, principalmente, para a prática cirúrgica precisa e segura. Tal compreensão torna-se particularmente essencial quando se trata de cirúrgica endoscópica e seu horizonte de recursos técnicos.

\section{REFERÊNCIASBIBLIOGRÁFICAS}

1. Cope VZ. The internal structure of the sphenoid sinus. J Anat (Lond) 1917:51:127-136.

2. Salinger S. The paranasal sinuses. Arch Otolaryng 1939;30:44.

3. Van Alyea OE. Nasal Sinuses: An Anatomic and Clinic Consideration. Baltimore: The Williams and Wilkins Company; 1951. p.155. 
4. Draf W. Endoscopy of Paranasal Sinuses. Berlin: Springer; 1983.

5. Fujii K, Chambers SM, Rhoton AL Jr. Neurovascular relationships of the sphenoid sinus: a microsurgical study. J Neurosurg 1979;50:31-39.

6. Van Alyea OE. Sphenoid sinus anatomic study with consideration of the clinical significance of the structural characteristics of the sphenoid sinus. Arch Otolaryng 1941;34:225-253.

7. Peele JC. Unusual anatomical variations of the sphenoid sinuses. Laryngoscope 1957;67:208-237.

8. Stammberger $H$. Results, problems, and complications. In: Stammberger H, Hawke M. Functional Endoscopic Sinus Surgery. The Messerklinger Technique. Philadelphia: BC Decker; 1991. p. 459-477.

9. Fahlbusch R, Buchfelder M. Present status of neurosurgery in the treatment of prolactinomas. Neurosurg Rev 1985;8:195-205.

10. Hammer G, Radberg C. Sphenoidal sinus: an anatomical and morfogenological study with reference to transsphenoid hypophysectomy. Acta Radiol (Stockh.) 1961;56:401-422.

11. Stankiewicz JA. Complications of endoscopic sinus surgery. Otolaryngol Clin North Am 1989;22:749-758.

12. Felippu A. Severe Epistaxis: The Transnasal Vessels Dissection. Instruction Course, Annual Meeting of the American Academy of Otolaryngology - Head and Neck Surgery: New Orleans; 1999

13. Maniglia AJ. Fatal and Major Complications Secondary to Nasal and Sinus Surgery. Laryngoscope 1989;99(3):276-283.

14. Lang J. In: Clinical Anatomy of the Nose Nasal Cavity and Paranasa Sinuses. New York: Thieme Medical Publishers; 1989. p.91.

15. Onodi A. The optic nerve and the accessory sinuses of the nose. New York: William Wood \& Co.; 1910.
16. Mellinger WJ. Optic nerves and their relatios in the spenoidal region. Tr. Pacific Coast Oto-Ophth Soc 1937;22:85.

17. Tunis JP. Sphenoidal sinusitis in relation to optic neuritis. Laryngoscope 1912;22:1157-1164.

18. Blum ME, Larson A. Mucocele of the sphenoidal sinus with sudden blindness. Layngoscope 1973;83:2024-2049.

19. Lang J, Keller H. Über die hintere Pfortenregion der Fossa pterygopalatina und das Ganglion pterigopalatinum. Gegenbaurs Morph Jb. 1978;124:207-214.

20.Zuckerkandl E. Normale und pathologische Anatomie der Nasenhöhle und ihrer pneumatischen Anhänge. $2^{\text {nd }}$ ed. Wien: W. Braumüller; 1893;1:368-400.

21. Sluder G. The role of the sphenopalatina (or Meckel's) ganglion in nasal headaches. Int Rec Med 1908;23:989-990.

22. Sluder G. In: Headaches and Eye Disorders of Nasal Origin. St. Louis: C. V. Mosby Co.; 1919.

23. Vail HH. Vidian Neuralgia. Ann Otol Rhin \& Laryng 1932;14:837.

24. Dixon FW. A comparative study of the sphenoid sinus (a study of 1600 skulls). Ann Otol 1937;46:687-698.

25. Mosher HP. The anatomy of the sphenoid sinus and the mothod of approching it from the antrum. Laryngoscope 1903;13:177-215.

26. Houser KM. Anatomic relations of the sphenoid sinus to Dorello's canal: abducens paralysis. Arch Otolaryng 1932;16:488.

27. Garber H. Sinusitis and neuralgia. Arch Otolaryng 1933;18:339.

28. Sluder G. Cited by Van Alyea.

29. Cope VZ. Internal structure of the sphenoid sinus. J Anat \& Physiol 1917;51:127.

30. Congdon ED. The distribution and mode of origin of septa and walls of the sphenoid sinus. Ant Rec 1920;18:97. 